TITLE:

\title{
Dynamics of order formation by colloidal adsorption onto a substrate studied with Brownian dynamics
}

AUTHOR(S):

Watanabe, S; Miyahara, M; Higashitani, K

\section{CITATION:}

Watanabe, S ... [et al]. Dynamics of order formation by colloidal adsorption onto a substrate studied with Brownian dynamics. JOURNAL OF CHEMICAL PHYSICS 2005, 122(10): 104704.

ISSUE DATE:

2005-03-08

URL:

http://hdl.handle.net/2433/39762

\section{RIGHT:}

Copyright 2005 American Institute of Physics. This article may be downloaded for personal use only. Any other use requires prior permission of the author and the American Institute of Physics. 


\title{
Dynamics of order formation by colloidal adsorption onto a substrate studied with Brownian dynamics
}

\author{
Satoshi Watanabe, Minoru Miyahara, ${ }^{\text {a) }}$ and Ko Higashitani \\ Department of Chemical Engineering, Kyoto University-Katsura, Nishikyo-ku, Kyoto 615-8510, Japan
}

(Received 28 July 2004; accepted 20 December 2004; published online 8 March 2005)

\begin{abstract}
Colloidal adsorption and spontaneous ordering of adsorbed particles on a substrate was simulated using a three-dimensional simulation model for colloidal dispersion system with an adsorptive surface under a specified bulk concentration, where the particle-particle and particle-substrate interactions were modeled on the DLVO theory. The key process for order formation is considered to be the adsorption of a particle that induces the transition from incomplete order to perfect order, and is found to involve a stochastic nature due to an energy barrier which must be overcome for the system to reach ordered state. Also, a model was developed to predict the energy barrier for order formation based on direct observation of the key process. Further, a model to describe the stochastic nature of the process was developed and its quantitative validity was demonstrated. Through the examination of the key process, it is concluded that the mechanism of the order formation is composed of two successive processes and the rate-determining step varies depending on the ionic strength. () 2005 American Institute of Physics. [DOI: 10.1063/1.1859279]
\end{abstract}

\section{INTRODUCTION}

Colloidal adsorption is an attractive process to fabricate a monolayer film on a substrate. Colloid particles are adsorbed onto a substrate with countercharge due to the electrostatic attraction, while the adsorbed particles repel particles in bulk because of the electrostatic repulsion when the surface coverage reaches saturation, which leads to the formation of a monolayer film. The main features of the colloidal adsorption are its rapidity, applicability to a wide range of particle size, and coverage control by changing the salt concentration or the thickness of the electric double layer. Thus the method would be suitable for various applications, such as antireflection coating, ${ }^{1}$ biosensors, ${ }^{2}$ data storage media, lithography masks, ${ }^{3}$ and catalysts, ${ }^{4}$ where the important particle size would be from hundred nanometers down to several nanometers. The quality of such products can be improved by the formation of a regular lattice structure of adsorbed particles, as seen in an example of antireflection coating. ${ }^{5}$ Furthermore, the order formation of adsorbed structures would open the possibility for the application to optical devices. In that sense, control of adsorbed structures is of great importance.

As for the formation of close-packed hexagonal arrays of particles, several techniques utilizing capillary forces acting between particles ${ }^{6}$ have been proposed, which include methods by drying particle suspension prepared as a thin wetting film on a substrate ${ }^{7}$ or by withdrawing a substrate from particle suspension. ${ }^{8}$ These approaches can produce closepacked arrays although they have difficulty in controling monolayer formation; a bilayer or multilayer may form in some areas. Colloidal adsorption has an advantage to cover large areas with a monolayer film of particles. Also, colloidal

\footnotetext{
${ }^{a)}$ Author to whom correspondence should be addressed. Electronic mail: miyahara@cheme.kyoto-u.ac.jp
}

adsorption has potential for fabricating ordered particle arrays with controlled surface coverage or interparticle spacing since the process contains important factors, such as repulsive characteristics among particles, control of interaction range by varying ionic strength, and the Brownian motion, which would be required to form ordered structures that are energetically more stable than random-packed structures.

Some experiments have studied structures of nanosized particles adsorbed on a substrate, observing polystyrene latex particles on a titanium oxide substrate, ${ }^{3}$ on a mica substrate, ${ }^{9-11}$ on an alumina substrate, ${ }^{12}$ and on a polymer film surface. ${ }^{13}$ Though a high degree of short-range order among particles was reported, no further and detailed analysis on adsorbed structures has been done. In addition to the observation of adsorbed structures, particle mobility at liquid-solid interface, i.e., an in-plain Brownian diffusion has also been studied for many combinations of particle and substrate, including latex particles on an alumina substrate, ${ }^{12}$ alumina colloids on mica, ${ }^{14,15}$ and gold colloids ${ }^{16}$ and glycoproteins ${ }^{17}$ on various substrates. No definitive conclusion, however, has been drawn and this issue is still under discussion because the particle mobility would depend on several factors such as geometric shape of particles, surface roughness of particles and substrates, and the adhesive force between particles and substrates, etc., of which it is difficult to make systematic studies on the effects. Nonetheless, those studies show at least the possibility for spherical particles to move along a smooth substrate, which leads to order formation.

Following the experimental studies, computer simulation studies on order-disorder phase transitions of adsorbed particle structures have been reported examining the following factors: the effect of particle potentials, wall potentials, particle concentration, and salt concentration onto orderdisorder boundaries, ${ }^{18}$ surface structures of charge-bidisperse 
particle mixtures, ${ }^{19}$ and the structural phase behavior of particles tethered to a substrate. ${ }^{20,21}$ Recently, we developed a three-dimensional cell model to simulate colloidal adsorption and order formation on a substrate. ${ }^{22}$ Our simulations demonstrated that the ordered structures of the adsorbed particles are formed only when what we call "one-directional average force" acting on an adsorbed particle exceeds a critical value, which is independent of the salt concentration or the interaction potentials. Though these studies clarified the dependence of wall and particle potentials on order-disorder transition and a determinant factor required for order formation, the kinetic process of adsorption and order formation has not been investigated.

In contrast to the physical adsorption of molecules, which attain equilibrium state quite easily, the colloidal adsorption exhibits highly nonequilibrium nature: surface coverage cannot attain a true equilibrium coverage because, as particle adsorption proceeds, an energy barrier due to the electrostatic repulsion between the adsorbed layer and a particle in bulk will form. Thus the energy barrier would play a crucial role not only in particle adsorption but also in the order formation, which needs a higher coverage. In most cases, nevertheless, the existence of the energy barrier has been qualitatively described only through the emergence of a depletion region between an adsorbed layer and bulk phase. ${ }^{18,23,24}$ As a quantitative description of the energy barrier, Antelmi and Spalla ${ }^{12}$ developed a model to calculate a potential-distance profile of a particle approaching a partially covered substrate as a function of surface coverage, but they did not consider adsorbed structures because of the employed assumption that adsorbed particles are fixed on a substrate. In order to model the kinetics of adsorption process, the authors think that a stochastic approach should be employed as seen in, for instance, the treatment of the nucleation process, ${ }^{25}$ which is typical of the processes involving an energy barrier. To our knowledge, no studies to date have examined the relationship of the energy barrier with structures of adsorbed particles on a substrate along this line.

In the present paper, we explore the kinetic process of particle adsorption and order formation by conducting Brownian dynamics simulations with the three-dimensional cell in which the particle-particle and particle-substrate interactions are modeled on the DLVO theory. ${ }^{26}$ The stochastic nature of the adsorption process is demonstrated by comparing several simulation runs with common conditions but with different initial configurations. We then focus on a particle that brings perfect order into the adsorbed phase upon its adsorption, and observe directly the behavior of the particle to reveal the mechanism of order formation. Through detailed analysis of the particle's adsorption process, we develop a stochastic model to describe the rate process of order formation taking into account the energy barrier. The quantitative validity of the model is demonstrated through comparison between the stochastic model and simulation results.

\section{MODEL AND METHOD}

The simulation cell is illustrated in Fig. 1. The solvent of the suspension, which is assumed to be water in the present

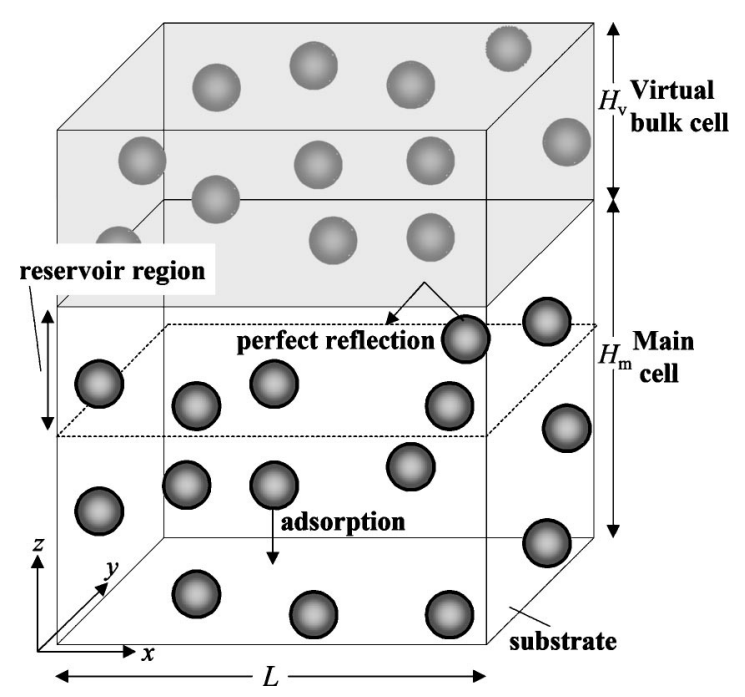

FIG. 1. A schematic drawing of the simulation cell.

study, has a viscosity $\eta$, a relative permittivity $\varepsilon$, and an inverse Debye length $\kappa$ that is proportional to the square root of ionic strength. The colloidal particles, which are modeled on polystyrene latex, have a radius $a$, a positive surface charge density $\sigma_{\mathrm{p}}$, and a diffusion coefficient $D$ given by the Stokes-Einstein relation $D=k_{\mathrm{B}} T / 6 \pi \eta a$, where $k_{\mathrm{B}}$ is the Boltzmann constant and $T$ is temperature. $\quad \psi_{\mathrm{p}}$ is the particle surface potential scaled by $k_{\mathrm{B}} T / e$, and is calculated under the constant charge condition with the following equation: ${ }^{27}$

$$
\frac{e \sigma_{\mathrm{p}}}{\kappa \varepsilon \varepsilon_{0} k_{\mathrm{B}} T}=\psi_{\mathrm{p}}+\frac{\psi_{\mathrm{p}}}{\kappa a}-\frac{\tau_{1}^{2} \kappa a}{\tau_{2}-\tau_{1} \kappa a},
$$

where $\tau_{1}=2 \sinh \left(\psi_{\mathrm{p}} / 2\right)-\psi_{\mathrm{p}}, \tau_{2}=4 \tanh \left(\psi_{\mathrm{p}} / 4\right)-\psi_{\mathrm{p}}, e$ is the elementary charge, and $\varepsilon_{0}$ is the vacuum permittivity of the solvent. The adsorptive substrate with a surface potential $\psi_{\mathrm{s}}$ is modeled on mica which has uniform negative charge.

A detailed description on the simulation cell is given in Ref. 22, and here we explain briefly the concept of the cell. The simulation cell is composed of two parts: the main cell and the virtual bulk cell. The former, which is periodic in the $x$ and $y$ directions, has an adsorptive surface at the bottom $(z=0)$ and a reflective boundary at the top $\left(z=H_{\mathrm{m}}\right)$. Since the particle-surface interaction is strong under the condition employed in the present study, the particle adsorption can be regarded as irreversible. In fact no desorption was observed within the simulation time. The virtual bulk cell, attached on the top boundary, is an independent bulk phase in which particle interactions follow the periodic boundary condition in all the directions. The particles in the main cell are assumed to be subjected to forces from the particles in the virtual bulk cell, and to exert no forces onto them; the relation between the two cells is "one-way." Without the virtual bulk cell, the particles in the main cell would localize in the vicinity of the top boundary because they would have no interaction with the top boundary. In addition, a reservoir region is set in the upper part of the main cell in order to keep the bulk concentration constant against adsorption of particles on the substrate, in which new particles are supplied if the concentration averaged over every 100 time step is less than the desired one. We have confirmed that the simulation 
TABLE I. Simulation parameters.

\begin{tabular}{|c|c|c|c|c|c|}
\hline$\kappa a[-]$ & 1 & 2 & 5 & 10 & 20 \\
\hline$L / a[-]$ & 88 & 66 & 44 & 36 & 30 \\
\hline$H_{\mathrm{v}} / a[-]$ & 24 & 14 & 8 & 6 & 5 \\
\hline$H_{\mathrm{m}} / a[-]$ & \multicolumn{5}{|c|}{20} \\
\hline$\phi(\mathrm{vol} / \mathrm{vol})$ & \multicolumn{5}{|c|}{$8.0 \times 10^{-4}-2.8 \times 10^{-1}$} \\
\hline$\Delta t(\mathrm{~ns})$ & \multicolumn{5}{|c|}{50} \\
\hline
\end{tabular}

cell solves the difficulty and makes it possible to simulate the colloidal dispersion system with an adsorptive substrate under a specified bulk concentration.

In a Brownian dynamics simulation, the motion of a colloidal particle $i$ in the suspension follows the discrete form of the Langevin equation: ${ }^{28}$

$$
\mathbf{r}_{i}(t+\Delta t)=\mathbf{r}_{i}(t)+D \frac{\mathbf{F}_{i}^{\mathrm{p}}}{k_{\mathrm{B}} T} \Delta t+\Delta \mathbf{r}_{i}^{\mathrm{B}}
$$

where $\mathbf{r}_{i}(t)$ is the position vector at time $t, \mathbf{F}_{i}^{\mathrm{p}}$ is the external force due to the particle-particle and particle-substrate interactions, $\Delta t$ is a time step, and $\Delta \mathbf{r}_{i}^{\mathrm{B}}$ is a random displacement which forms a Gaussian distribution with $\left\langle\Delta \mathbf{r}_{i}^{\mathrm{B}}(t)\right\rangle=\mathbf{0}$ and $\left\langle\left(\Delta r_{i, k}^{\mathrm{B}}(t)\right)\left(\Delta r_{j, l}^{\mathrm{B}}(t)\right)\right\rangle=2 D \Delta t \delta_{i j} \delta_{k l}$, where $k$ and $l$ are indices for the coordinate directions $(x, y, z)$, and $\delta_{i j}$ is the Kronecker delta function.

The external force $\mathbf{F}_{i}^{\mathrm{p}}$ is calculated based on the DLVO theory. The electrostatic and the van der Waals interactions, $E_{\mathrm{el}}$ and $E_{\mathrm{vdW}}$, respectively, are given by the following equations $^{23,27}$ scaled by $k_{\mathrm{B}} T$ :

$$
E_{\mathrm{el}(\mathrm{pp})}=\left(\frac{4 \pi k_{\mathrm{B}} T \varepsilon \varepsilon_{0} a}{e^{2}}\right)\left(\frac{\psi_{\mathrm{p}}+4 \gamma \Omega \kappa a}{1+\Omega \kappa a}\right)^{2} \frac{1}{r} \exp [-\kappa a(r-2)],
$$

$$
E_{\mathrm{vdW}(\mathrm{pp})}=-\frac{A_{\mathrm{pp}}}{6 k_{\mathrm{B}} T}\left[\frac{2}{r^{2}-4}+\frac{2}{r^{2}}+\ln \left(1-\frac{4}{r^{2}}\right)\right]
$$

$E_{\mathrm{el}(\mathrm{ps})}=\left(\frac{4 \pi k_{\mathrm{B}} T \varepsilon \varepsilon_{0} a}{e^{2}}\right)\left(\frac{\psi_{\mathrm{p}}+4 \gamma \Omega \kappa a}{1+\Omega \kappa a}\right)\left[4 \tanh \left(\frac{\psi_{\mathrm{s}}}{4}\right)\right]$

$$
\times \exp [-\kappa a h] \text {, }
$$

$E_{\mathrm{vdW}(\mathrm{ps})}=-\frac{A_{\mathrm{ps}}}{6 k_{\mathrm{B}} T}\left[\frac{1}{h}+\frac{1}{h+2}+\ln \left(\frac{h}{h+2}\right)\right]$,

where the subscript pp means particle-particle and ps particle-substrate. $r$ and $h$ are the dimensionless center-tocenter interparticle distance and the dimensionless surfaceto-surface separation distance between a particle and a substrate, both of which are scaled by $a$. A is Hamaker's constant, and $\gamma$ and $\Omega$ are given by $\gamma=\tanh \left(\psi_{\mathrm{p}} / 4\right)$ and $\Omega$ $=\left(\psi_{\mathrm{p}}-4 \gamma\right) /\left(2 \gamma^{3}\right)$.

The sum of the two contributions, $E_{\mathrm{el}}$ and $E_{\mathrm{vdW}}$ gives the total particle-particle and particle-substrate interactions. The particle-particle interaction is repulsive, while the particlesubstrate interaction is attractive under the condition employed in the present study.

The simulation condition is described in Ref. 22, and here we summarize the simulation parameters and the physi-
TABLE II. Physical parameters.

\begin{tabular}{ccc}
\hline \hline$a$ & $(\mathrm{~nm})$ & 50 \\
$\sigma_{\mathrm{p}}$ & $\left(\mathrm{mC} / \mathrm{m}^{2}\right)$ & 27.4 \\
$\psi_{\mathrm{s}}$ & $(\mathrm{mV})$ & -100 \\
$D$ & $\left(\mathrm{~m}^{2} / \mathrm{s}\right)$ & $4.88 \times 10^{-2}$ \\
$A_{\mathrm{pp}}$ & $(\mathrm{J})$ & $0.95 \times 10^{-20}$ \\
$A_{\mathrm{ps}}$ & $(\mathrm{J})$ & $1.60 \times 10^{-20}$ \\
$T$ & $(\mathrm{~K})$ & 298 \\
$\eta$ & $(\mathrm{Pa} \cdot \mathrm{s})$ & $8.94 \times 10^{-4}$ \\
$\varepsilon$ & {$[-]$} & 78.3 \\
\hline \hline
\end{tabular}

cal properties in Tables I and II, respectively. The system size we employed is comparable to that of Ref. 18, and further, we confirmed that the simulations with twice the side length yielded no significant difference of structural results. The dimensionless product $\kappa a$, the ratio of the radius of a particle to the Debye length, is an important parameter that determines the range of the electrostatic interaction. The volume fraction of the particles in the bulk phase $\phi$ is another important factor to be examined.

\section{RESULTS AND DISCUSSION}

\section{A. Probabilistic nature in adsorption process}

Figure 2 shows the time evolution of surface coverage $\theta$ for five simulation runs with common $\kappa a$ and $\phi(\kappa a=5$ and $\phi=0.08$ ), among which only initial configurations of particles are different. Surface coverages for five runs show quite different behavior, except for the very beginning of the adsorption process. As surface coverages increase, the differences stand out; three of the five runs (runs 1, 3, and 4) reach the critical surface coverage $\theta^{c}$ for order formation, i.e., attain the ordered state within the simulation time, but the rest (runs 2 and 5) do not. The critical surface coverage $\theta^{c}$ for various $\kappa a$ was obtained in our previous work ${ }^{22}$ as summarized in the first row of Table III. Although runs 2 and 5 might reach the ordered state in some time, it would be difficult to know the time. This behavior reflects a stochastic nature of the adsorption process of particles, and suggests that it is almost impossible to describe in a deterministic way the time evolution of surface coverage or to predict the time needed to reach the ordered state. Since the stochastic nature becomes marked when the surface coverage comes close to the critical one, it would be attributed to an energy barrier

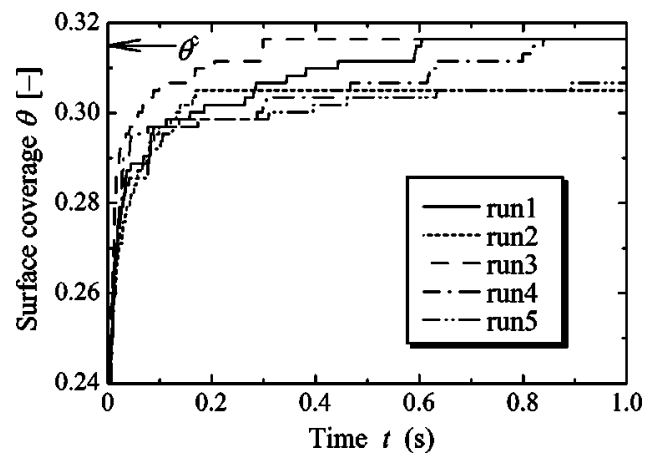

FIG. 2. Time evolution of the surface coverage for five different runs in which only initial configurations are different at $\kappa a=5$ and $\phi=0.08$. 
TABLE III. Values of $\theta^{c}, l^{c}, V_{\mathrm{ad}}$, and $V_{\mathrm{d}}$ for five values of $\kappa a$.

\begin{tabular}{cccccc}
\hline \hline$\kappa a[-]$ & 1 & 2 & 5 & 10 & 20 \\
\hline$\theta^{c}[-]$ & $0.787 \times 10^{-1}$ & 0.156 & 0.315 & 0.456 & 0.576 \\
$l^{c}(\mathrm{~nm})$ & 332 & 242 & 170 & 142 & 124 \\
$V_{\mathrm{ad}}\left(k_{\mathrm{B}} T\right)$ & 0.35 & 4.78 & 10.8 & 10.8 & 10.2 \\
$V_{\mathrm{d}}\left(k_{\mathrm{B}} T\right)$ & 8.6 & 11.6 & 6.50 & 3.82 & 2.28 \\
\hline \hline
\end{tabular}

built up by adsorbed particles. Particles in bulk phase have to overcome the barrier to get adsorbed onto a substrate covered with many adsorbed particles.

If the adsorption process of particles is stochastic, the process of order formation must also be a stochastic phenomenon. The key process for having the ordered state would be the last adsorption that brings the transition from the structure with incomplete order to that with perfect order. In order to see the probabilistic nature of the key process in detail, a single simulation run would not be sufficient. Hence, we sampled the elapsed time required for the last adsorption by conducting 200 simulation runs of the following simulation: each of the simulation runs started from a snapshot of adsorbed particles before the last adsorption with a different random seed for Brownian motion. The bulk particles were placed randomly with a given concentration. We prepared four initial configurations of adsorbed particles for a given condition of $\kappa a$ and $\phi$, and carried out 50 simulation runs for each initial configuration, which produced 200 samples of the time needed for the last adsorption or the order formation. The results are expressed as the probability $P(t)$ for remaining disordered after a time $t$. Following the definition, $P\left(t_{\mathrm{a}}\right)=0.2$, e.g., means that $80 \%$ of samples have got ordered before $t=t_{\mathrm{a}}$. Figure 3(a) shows $P(t)$ for $\kappa a=5$ with various
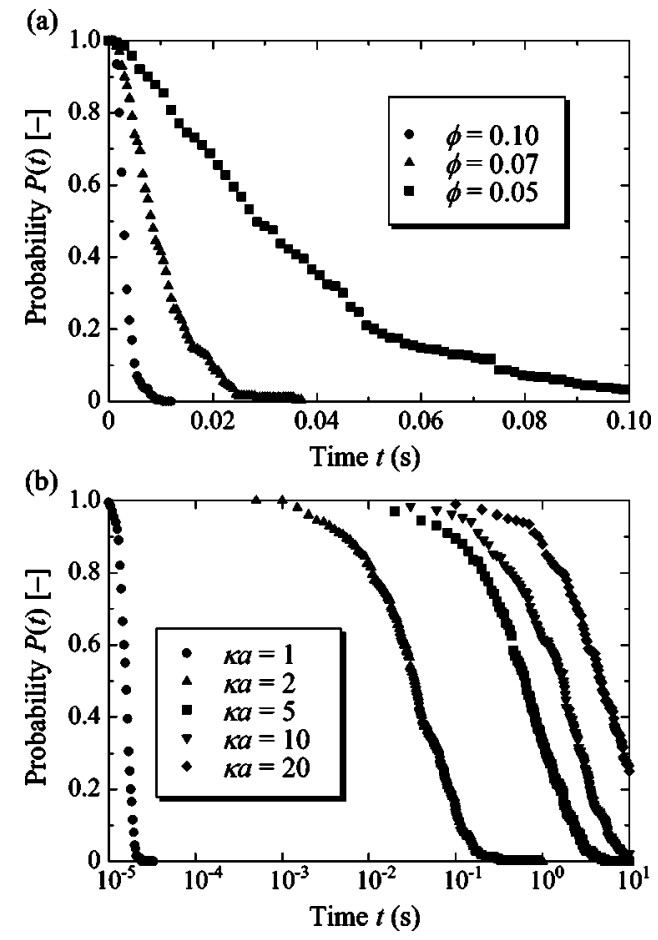

FIG. 3. Probability of remaining disordered state for (a) $\kappa a=5$ with various values of bulk concentration $\phi$ and (b) various values of $\kappa a$ with $\phi=0.01$.

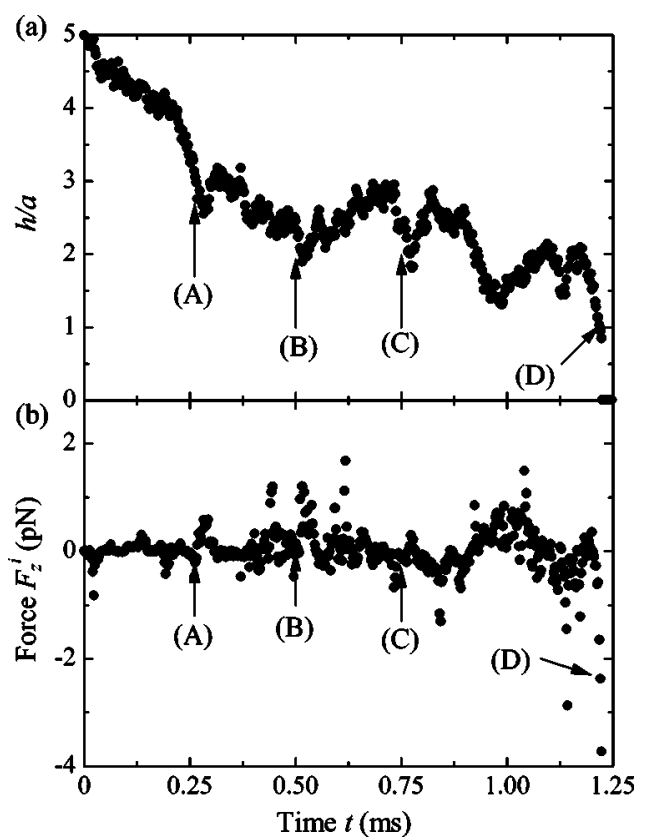

FIG. 4. The adsorption process of the last particle for $\kappa a=5$ and $\phi=0.07$ : (a) height variation, (b) force profile in $z$ direction. Indications (A)-(D) correspond to the snapshots of Fig. 5.

values of $\phi . P(t)$ exhibits an exponential-type decay against the elapsed time, and thus the process of the last adsorption clearly demonstrates the characteristics of stochastic phenomena. Also, $P(t)$ depends significantly on the bulk concentration; the distribution of the elapsed time becomes wide as the bulk concentration decreases. This behavior would be qualitatively reasonable because decreasing the bulk concentration leads to the decrease both in the bulk potential that would push a particle to the substrate and in the frequency of the attempts for particles' adsorption onto the substrate.

The effect of $\kappa a$ on $P(t)$ is shown in Fig. 3(b) and is more significant than that of the bulk concentration. For $\kappa a$ $=1$ the last adsorption occurs in tens of microseconds, and as $\kappa a$ increases, the time required for the adsorption increases several orders of magnitude. Since the particle-substrate attractive interaction becomes strong and long ranged as $\kappa a$ decreases, the increase in the particle-substrate attraction would lower an energy barrier, resulting in easy and fast adsorption. Although the particle-particle repulsion increases with decreasing $\kappa a$, the particle-substrate attraction would play a dominant role in the adsorption process, prevailing against the increase in the particle-particle repulsion.

In order to have a quantitative understanding of the effects of the interactions and the bulk concentration onto the last adsorption process, it would be necessary to investigate the detailed mechanism of the key process by directly observing the last adsorption, which is described in the following section.

\section{B. Dynamics of the last adsorption}

We observed directly the order formation process induced by the last adsorption for various conditions of $\kappa a$ and $\phi$. As a typical example of the process, Figs. 4 and 5 show the case for $\kappa a=5$ and $\phi=0.07$. Figures 4(a) and 4(b) show 

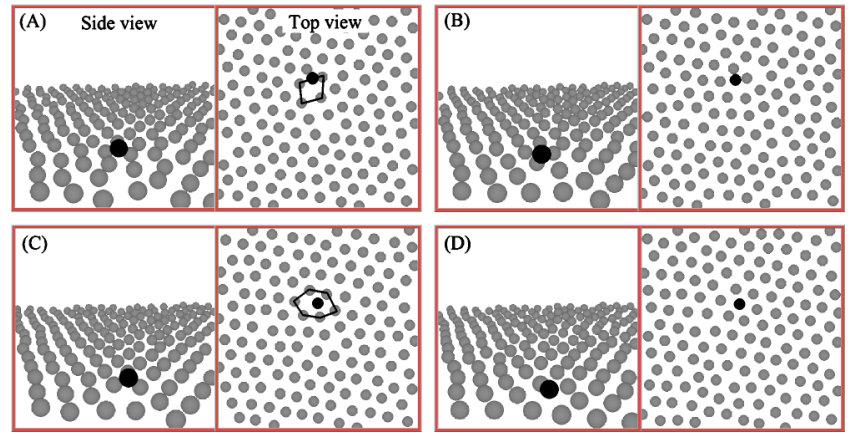

FIG. 5. Snapshots of the adsorption process of the last particle for $\kappa a=5$ and $\phi=0.07$.

the variation of height $h$ and the force $F_{z}^{i}$ in $z$ direction acting on the last particle labeled $i$ against time during the adsorption process, where $t=0$ is set at the moment the observation was started. Figures 5(A)-5(D) show snapshots taken at time pointed by the corresponding arrow in Figs. 4(a) and 4(b), in which the last particle $i$ is drawn in black, the adsorbed particles are in gray, and the rest of the particles in the suspension are not shown for clarity. These figures demonstrate the detailed adsorption process. The particle $i$ comes down to a relatively disordered part where particles form almost a square arrangement rather than a triangle [Fig. 5(A)], approaches gradually near to the substrate fighting against repulsive forces from the particles in the disordered part [Fig. $5(\mathrm{~B})]$, and then is repelled away by them. The particle $i$, however, makes some attempts at adsorption at around $t=0.50,0.75,0.90$, and $1.12 \mathrm{~ms}$. After the failures, the particle $i$ pushes the particles of the squarelike part aside until they form almost a hexagonal arrangement [Fig. 5(C)]. Finally the particle $i$ gets adsorbed into the center of the hexagon [Fig. 5(D)]. Here it is interesting that not one but some disordered parts are seen in Figs. 5(A) and 5(B) even though the coverage is quite close to the critical surface coverage $\theta^{c}$, and the adsorption of the last particle alters the other disordered parts into the hexagonal arrangement. Though not shown here similar processes of the last adsorption were observed in the cases for larger values of $\kappa a, 10$ and 20.

As an example of the case for a smaller value of $\kappa a$, the observation results for $\kappa a=1$ and $\phi=0.001$ are shown in Figs. 6 and 7, which are in the same format as Figs. 4 and 5. It is clearly seen that a disordered squarelike part is made after the approach of the particle $i$ to a triangle arrangement in contrast to the cases with large $\kappa a$, in which the disordered part was existing before the $i$-particle's approach. The particle $i$ approaches gradually to the adsorbed phase [Fig. 7(A)], and wanders above a triangle being subjected to repulsive forces from the already adsorbed particles [Fig. 7(B)]. Then the particle $i$ begins to penetrate the triangle [Fig. $7(\mathrm{C})]$, changing the triangle arrangement into a squarelike one [Fig. 7(D)], and finally gets adsorbed. In this last stage the adsorption into a squarelike part proceeds so fast that the squarelike part does not have sufficient time to transform itself into a hexagonal arrangement contrary to the case for larger $\kappa a$. After the adsorption, however, the struc-

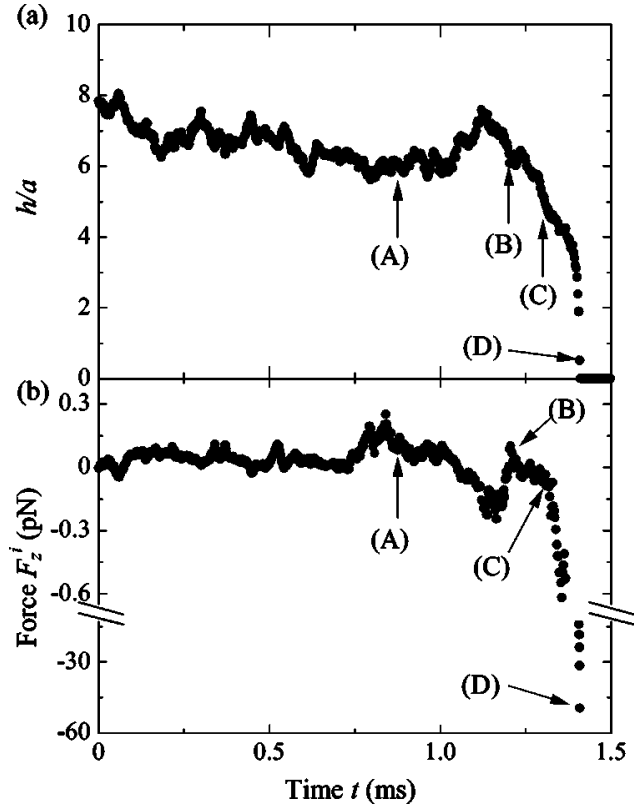

FIG. 6. The adsorption process of the last particle for $\kappa a=1$ and $\phi=0.001$ : (a) height variation, (b) force profile in $z$ direction. Indications (A)-(D) correspond to the snapshots of Fig. 6.

tural rearrangement of the part continues until the part turns into a hexagon. Similar manner of order formation was observed in the case for $\kappa a=2$.

Figure 8 shows typical force-distance profiles of the last particle $i$ for $\kappa a=5$ and $\kappa a=1$, in which only the maximum value of the force $F_{z}^{i}$ at each distance $h$ is plotted for clarity. The last particle for both values of $\kappa a$ must go through repulsive forces in order to get adsorbed. However, the repulsive forces come from different processes depending on the $\kappa a$. For $\kappa a=5$, repulsive forces at $h / a=1.5-2.0$ come from the process of pushing the particles of a disordered squarelike structure aside to get adsorbed [Figs. 5(B) and 5(C)]. On the other hand, for $\kappa a=1$ the repulsion around $h / a=5-8$ is due to the process of trying to disturb a triangle structure [Figs. 7(A)-7(C)].

The above observation reveals that the process of the order formation involves the disturbance process to make a disordered squarelike part and the adsorption process onto a disordered squarelike part: the two processes are successive. The mechanism is as follows. For larger $\kappa a(5,10$, and 20), it would be easy and fast to disturb triangle structures. A
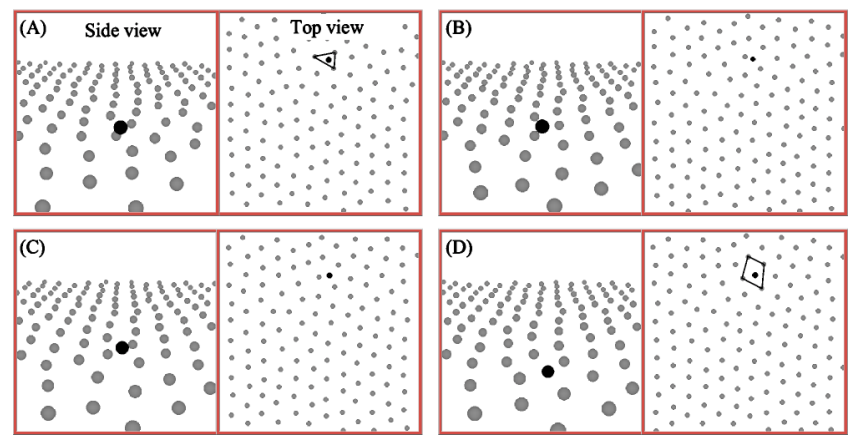

FIG. 7. Snapshots of the adsorption process of the last particle for $\kappa a=1$ and $\phi=0.001$. 
(a)
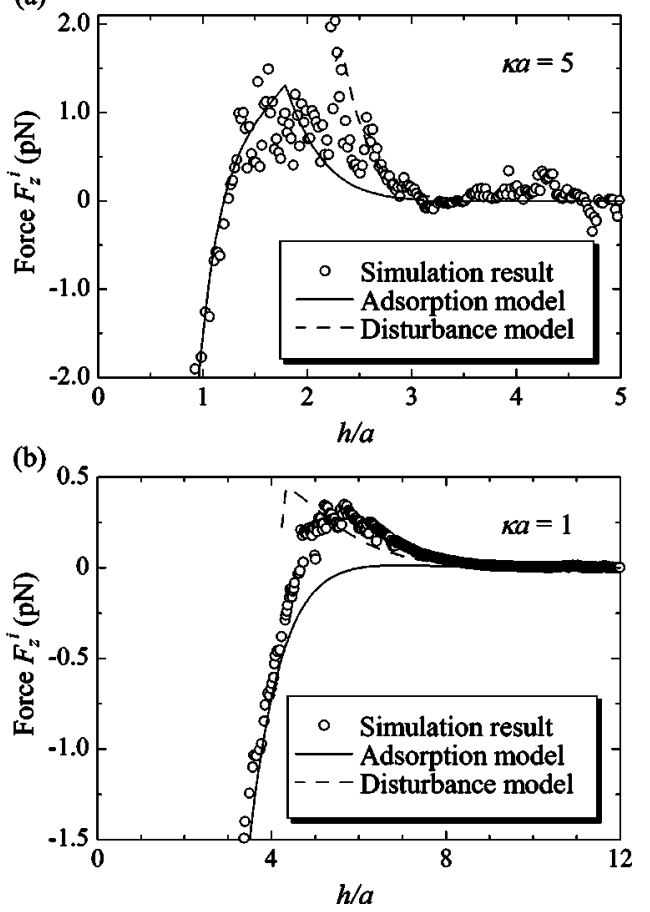

FIG. 8. Force-distance profile of the last particle obtained from the simulation and the proposed models: (a) $\kappa a=5$, and (b) $\kappa a=1$.

disordered part may be made up by other particles than the last particle, given that some disordered parts are observed. For smaller $\kappa a$ (1 and 2), in contrast, the disturbance process is much more time consuming than the adsorption process, and necessarily a particle that happens to make a disordered part would become the last adsorption particle.

Then an important factor for order formation would be the potential energy acting on the last particle since the magnitude of the energy barrier determines the probability of the adsorption. Now the potential energy is estimated by modeling the order formation process as described in the following section.

\section{Modeling of order formation process}

The modeling is based on a characteristic property of the order formation of adsorbed particles: in our previous work, ${ }^{22}$ we examined intensively the condition required for order formation of adsorbed particles on a substrate, and found that the determinant factor for order formation, which is common to any value of $\kappa a$, is neither the potential energy in adsorbed phase nor the two-dimensional pressure of the adsorbed particles, but "the one-directional average force" acting on an adsorbed particle $F_{\text {av }}$ defined by the following equation:

$$
F_{\mathrm{av}}=\frac{1}{N_{\mathrm{s}}}\left\langle\sum_{i=1}^{N_{\mathrm{s}}}\left(\frac{\left|F_{x+}^{i}\right|+\left|F_{x-}^{i}\right|+\left|F_{y+}^{i}\right|+\left|F_{y-}^{i}\right|}{4}\right)\right\rangle,
$$

where $N_{\mathrm{s}}$ is the number of the adsorbed particles and $F_{x+}^{i}$ is the sum of the $x$-component forces on particle $i$ acting from particles $j$ existing on the right-hand side of it, i.e., satisfying $x_{j}>x_{i}$. Thus the four terms of $F^{i}$ in the numerator means that the forces of all four directions are averaged. The ordered
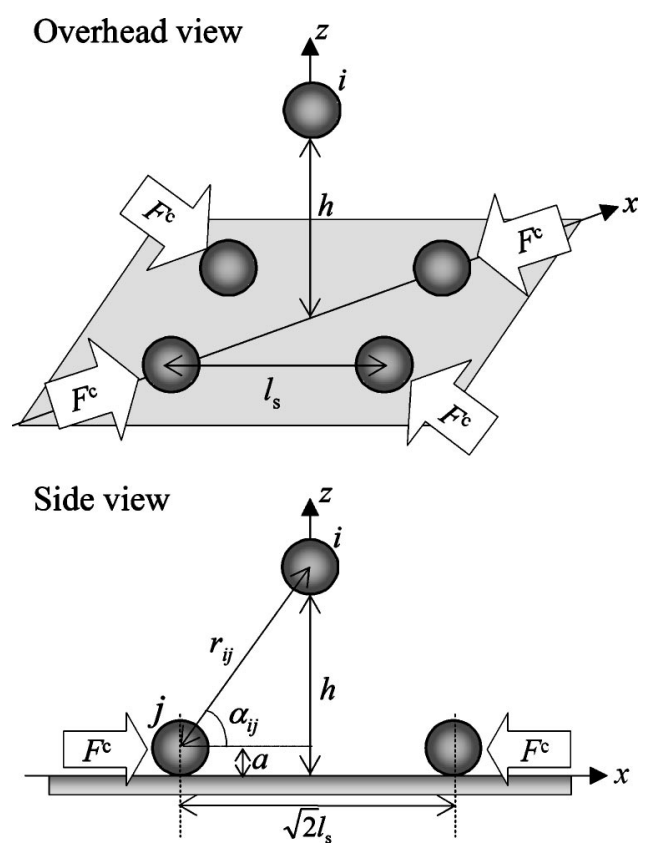

FIG. 9. A schematic drawing of the adsorption process model.

structures appear only when $F_{\text {av }}$ exceeds a critical value $F^{\mathrm{c}}$, or in other words, adsorbed particles in the ordered structures are subjected to the critical force $F^{\mathrm{c}}$ in any direction, regardless of the values of $\kappa a$.

We model the order formation process assuming that adsorbed particles at the coverage just below the critical surface coverage are subjected to the critical force. First, we calculate the energy barrier for the adsorption of a last particle, or for the order formation (Sec. III C 1), and then, describe the stochastic phenomena of the order formation utilizing the obtained energy barrier (Sec. III C 2).

\section{Estimation of the energy barrier for order formation}

The adsorption process of the last particle onto a disordered squarelike part is modeled as illustrated in Fig. 9. The scheme of the model is as follows.

(i) A square structure with an initial side length $l_{\mathrm{s}}$ composed of four adsorbed particles labeled $j(j=1-4)$ is set on a substrate, where the adsorbed particles are assumed to be subjected to the critical average force $F^{\mathrm{c}}$.

(ii) A particle $i$ approaching to the substrate is set above the center of the square with the particle surfacesubstrate distance $h$.

(iii) Upon approach a force-distance curve of the particle $i$ as a function of $h$ is calculated based on the following scheme considering the balance between the critical average force $F^{\mathrm{c}}$ and the horizontal component of the force acting on an adsorbed particle $j, F_{x y}^{j}$.

(a) If $F_{x y}^{j} \leqslant F^{\mathrm{c}}$, the adsorbed particles $j$ stay at initial positions, i.e., $l=l_{\mathrm{s}}$.

(b) If $F_{x y}^{j}>F^{\mathrm{c}}$, the adsorbed particles are moved, or the square is expanded to have a side length $l$ that satisfies the equation $F_{x y}^{j}=F^{\mathrm{c}}$. With slightly smaller $h$ this calculation is repeated: in other 
words, the square is gradually expanded maintaining the force $F^{\mathrm{c}}$ with surroundings. The force-distance relation will be given by Eq. (11) for each distance $h$.

(iv) By integrating the calculated force-distance curve, the potential energy curve for the particle $i$ is obtained. As for the initial side length $l_{\mathrm{s}}$, detailed examination of the structures and pair correlation functions revealed that particle-particle distances of disordered squarelike parts are by $5 \%$ longer than the nearest particle-particle distance $l^{\mathrm{c}}$ of the ordered structure at $\theta=\theta^{c}$, and accordingly the initial side length of the square $l_{\mathrm{s}}$ is set as $1.05 \times l^{\mathrm{c}}$. The values of $l^{\mathrm{c}}$ are summarized in the second row of Table III. Here it is noted that the value of $l^{\mathrm{c}}$ and $\theta^{\mathrm{c}}$ can be calculated analytically from the value of the critical average force $F^{\mathrm{c}}$ based on the particle-particle interaction force: ${ }^{22}$

$$
\begin{aligned}
l^{\mathrm{c}}= & \frac{1}{\kappa} \ln \left[\frac{C}{\left.\ln \left(\frac{C}{(\ln 2 C)^{2}}+\frac{C}{\ln 2 C}\right)\right\}^{2}}\right. \\
& \left.+\frac{C}{\ln \left(\frac{C}{(\ln 2 C)^{2}}+\frac{C}{\ln 2 C}\right)}\right]
\end{aligned}
$$

with

$$
\begin{aligned}
C= & \frac{2+\sqrt{3}}{2} \frac{1}{F^{\mathrm{c}}}\left(\frac{4 \pi k_{\mathrm{B}} T \varepsilon \varepsilon_{0}}{e^{2}}\right) \\
& \times\left(\frac{\psi_{\mathrm{p}}+4 \gamma \Omega \kappa a}{1+\Omega \kappa a}\right)^{2}(\kappa a)^{2} \exp (2 \kappa a) .
\end{aligned}
$$

Then $\theta^{c}$ is given by

$$
\theta^{c}=\frac{\pi}{2 \sqrt{3}}\left(\frac{a}{l^{\mathrm{c}} / 2}\right)^{2} \text {. }
$$

Considering the geometric condition shown in Fig. 9, the force acting on the particle $i$ in $z$ direction, $F_{z}^{i}$, and the horizontal component of a particle $j, F_{x y}^{j}$, are given by the following equations:

$$
\begin{aligned}
& F_{z}^{i}(h, l)=4\left\{\left(-\frac{\mathrm{d} E_{\mathrm{pp}}\left(r_{i j}\right)}{\mathrm{d} r_{i j}}\right) \sin \alpha_{i j}\right\}-\frac{\mathrm{d} E_{\mathrm{ps}}(h)}{\mathrm{d} h}, \\
& F_{x y}^{j}(h, l)=\left(-\frac{\mathrm{d} E_{\mathrm{pp}}\left(r_{i j}\right)}{\mathrm{d} r_{i j}}\right) \cos \alpha_{i j},
\end{aligned}
$$

where $\alpha_{i j}$ is the angle of the line between particles $i$ and $j$ from the $x$ axis, and $r_{i j}$ is the distance between the centers of particles $i$ and $j$. Note that the distance $r_{i j}$ is the function of $h$ and $l$ and is expressed for a given $h$ as follows: (a) If $F_{x y}^{j}\left(h, l_{\mathrm{s}}\right) \leqslant F^{\mathrm{c}}, r_{i j}=\sqrt{h^{2}+l_{\mathrm{s}}^{2} / 2}$, (b) If $F_{x y}^{j}\left(h, l_{\mathrm{s}}\right)>F^{\mathrm{c}}$, an extended side length $l$ is first calculated by an iterative calculation so that $l$ satisfies $F_{x y}^{j}(h, l)=F^{\mathrm{c}}$ with $r_{i j}=\sqrt{h^{2}+l^{2} / 2}$, and then the obtained $r_{i j}$ is used in Eq. (11).
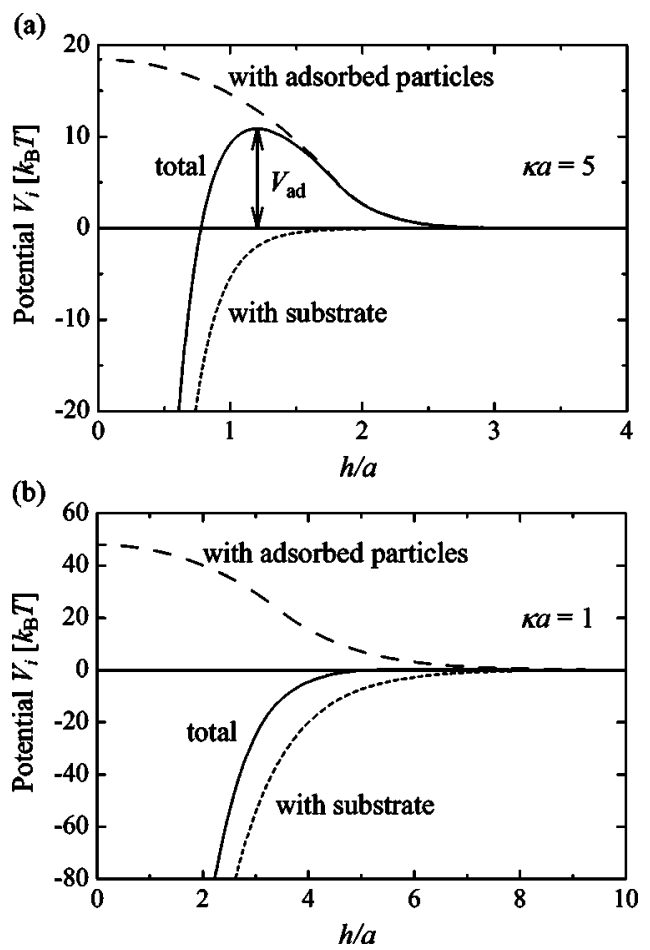

FIG. 10. Potential curves obtained from the adsorption process model for (a) $\kappa a=5$ and (b) $\kappa a=1$.

To check the validity of the model, the force-distance curves obtained from the above-explained adsorption process model are shown as a solid line in Fig. 8 comparing with the force-distance data of the last particle obtained from the simulation. As seen, the force-distance curve for $\kappa a=5$ is in good accordance with the simulation result. Contrarily, the case for $\kappa a=1$ does not give agreement with the adsorption process model, but it is still quite reasonable because the rate-determining step for small $\kappa a$ is not the adsorption process but the disturbance process as explained in Sec. III B, which will be quantitatively modeled later in this section. The agreement between the adsorption model and the simulation with lager $\kappa a$ demonstrates the model's quantitative performance. While the present model of the adsorption process is quite simple and considers only extension of the square structure caused by the approach of the particle $i$, it can grasp the essential point of the last adsorption process well.

By integrating the calculated force-distance curve, the potential energy curve for the last particle is obtained as shown in Figs. 10(a) and 10(b). The total potential energy, which is the sum of the potentials with already adsorbed particles and with the substrate, has an energy barrier $V_{\mathrm{ad}}$, which must be overcome by the particle getting adsorbed onto a disordered part to make the structure ordered. Figures 10 (a) and 10(b) show quite good contrast; for $\kappa a=5$ the energy barrier is clearly seen, but for $\kappa a=1$ no distinct barrier. The values of $V_{\text {ad }}$ for various $\kappa a$ are summarized in Table III. For $\kappa a=5,10$, and $20, V_{\text {ad }}$ is about $10 k_{\mathrm{B}} T$. Considering that colloidal particles are generally said to be dispersed in a stable state if the interparticle energy barrier exceeds $(10-15) k_{\mathrm{B}} T$, the obtained value $10 k_{\mathrm{B}} T$ seems to be in reasonable range. On the other hand, for $\kappa a=1$ and $2, V_{\text {ad }}$ is much 
smaller and would not serve as a "barrier." As expected from the observation results (Sec. III B), for $\kappa a=1$ and 2, the disturbance process to make a disordered part would be the rate-determining process, and should be modeled to estimate the energy barrier for the order formation.

The disturbance process, however, is rather complicated and difficult to model since the process involves the structure change from a triangle to a square. We thus simplify the process: the change of the arrangement is neglected and only the extension of the sides of a triangle arrangement is considered in the way similar to the case for the adsorption process model. We set a regular triangle structure with the side length $l^{\mathrm{c}}$ of three particles on a substrate and a particle $i$ above the center of the triangle, where the particles on a substrate are assumed to be subjected to the critical average force $F^{\mathrm{c}}$. Then we calculate the force acting on the particle $i$ approaching to the center of the triangle until the particle $i$ elongates the side length of the triangle from $l^{\mathrm{c}}$ to the length of the square structure $l_{\mathrm{s}}$. The adsorbed particles move only when the particle $i$ exerts the force with its horizontal component larger than $F^{\mathrm{c}}$, which is the same condition applied in the adsorption process model.

The calculated force-distance curves of the disturbance process are given also in Fig. 8 as a broken line, which demonstrates that the disturbance model expresses well the rising part of the force profile observed in the simulation $(h / a=2 \sim 3$ for $\kappa a=5$ and $h / a=5 \sim 8$ for $\kappa a=1)$.

Then the energy to elongate the side length from $l^{\mathrm{c}}$ to $l_{\mathrm{s}}$ is obtained by integrating the calculated force-distance curve. The obtained potential energy $V_{\mathrm{d}}$ for five different values of $\kappa a$ is summarized in Table III. In contrast to the result of the adsorption process, $V_{\mathrm{d}}$ for $\kappa a=1$ and 2 is about $10 k_{\mathrm{B}} T$ and larger than that for $\kappa a=5,10$, and 20 .

The above results for the energy barrier for the order formation supports the mechanism derived from the observation: for smaller $\kappa a$ the rate-determining step is the disturbance process rather than the adsorption process, and for the larger $\kappa a$, on the other hand, the adsorption onto a disordered part is the dominant process. Consequently, it is probable that the mechanism of the order formation is a successive process composed of the disturbance and adsorption steps, and the rate-determining process alters depending on the values of $\kappa a$.

\section{Rate process for order formation}

Since we obtained the energy barrier for the order formation, we can now consider the probabilistic nature of the order formation process seen in Figs. 3(a) and 3(b). Assuming that the process is a sequential process composed of the disturbance process and the adsorption process, the probability $P(t)$ of remaining in nonordered state at $t=t$ is given by

$$
P(t)=P_{3}+P_{4},
$$

where $P_{3}$ is the probability for remaining in triangle structures and $P_{4}$ is that for being square structures. Let us define the rate constant $c_{3}$ and $c_{4}$ as measures of easiness for the disturbance process and the adsorption process, respectively.
Then a schematic diagram for the order formation process is drawn in terms of the probability as follows:

$$
P_{3} \stackrel{c_{3}}{\rightarrow} P_{4} \stackrel{c_{4}}{\longrightarrow}[\text { ordered state }]
$$

In the diagram, triangle structures decrease with the rate $c_{3}$, and squares increase by the decreased amount of triangles and decrease with the rate $c_{4}$ :

$$
\begin{aligned}
& \frac{\mathrm{d} P_{3}}{\mathrm{~d} t}=-c_{3} P_{3}, \\
& \frac{\mathrm{d} P_{4}}{\mathrm{~d} t}=c_{3} P_{3}-c_{4} P_{4} .
\end{aligned}
$$

Then $P(t)$ is given by solving Eqs. (12) and (13):

$$
P(t)=\frac{1}{c_{4}-c_{3}}\left\{c_{4} \exp \left(-c_{3} t\right)-c_{3} \exp \left(-c_{4} t\right)\right\} .
$$

Following the definition of the rate constants, $c_{3}$ and $c_{4}$ must be proportional to the frequency of the particles' overcoming of the energy barrier $V_{\mathrm{d}}$ and $V_{\mathrm{ad}}$, respectively. Considering the net energy barrier for a particle in bulk phase with a certain potential energy $V_{\mathrm{b}}$, the frequency $J_{3}$ for the disturbance process can be expressed as

$$
J_{3}=n_{\mathrm{b}} \exp \left(\frac{V_{\mathrm{d}}-V_{\mathrm{b}}}{k_{\mathrm{B}} T}\right) \nu^{\mathrm{B}}\left(1-\theta^{\mathrm{c}}\right) L^{2},
$$

where $n_{\mathrm{b}}$ is number density of particles in bulk, $\left(V_{\mathrm{d}}-V_{\mathrm{b}}\right)$ is the net energy barrier for a particle to overcome the barrier for disturbing a triangle structure, $\nu^{\mathrm{B}}$ is the velocity of the particle due to Brownian random force, and $\left(1-\theta^{c}\right) L^{2}$ expresses the area of triangle structures excluding adsorbed particles where particles in bulk can approach. All the ordered triangle structures are taken into account as the site to be disturbed in determining the frequency $J_{3}$, and accordingly, $c_{3}$ is equal to $J_{3}$.

Similarly to $J_{3}$, the frequency for the adsorption process, $J_{4}$, is given by the following equation:

$$
J_{4}=n_{\mathrm{b}} \exp \left(\frac{V_{\mathrm{ad}}-V_{\mathrm{b}}}{k_{\mathrm{B}} T}\right) \nu^{\mathrm{B}} S
$$

where $S\left(=l_{\mathrm{s}}^{2}\right)$ means the area of the square structure of the disordered part shown in Fig. 9. Here it should be noted that $J_{4}$ is the frequency for particles to get adsorbed onto a single disordered square structure. However, as mentioned above, some disordered parts are observed in the structures near ordered state, and accordingly $c_{4}$ should be proportional to the frequency $J_{4}$ with a factor $c_{4}^{\prime}$ which means the number of disordered parts, as $c_{4}=c_{4}^{\prime} J_{4}$.

Further, since the two important factors, the bulk potential $V_{\mathrm{b}}$ and the velocity $\nu^{\mathrm{B}}$, exhibit certain distributions, the integral average over each of the distributions should be taken. Thus finally we obtain 


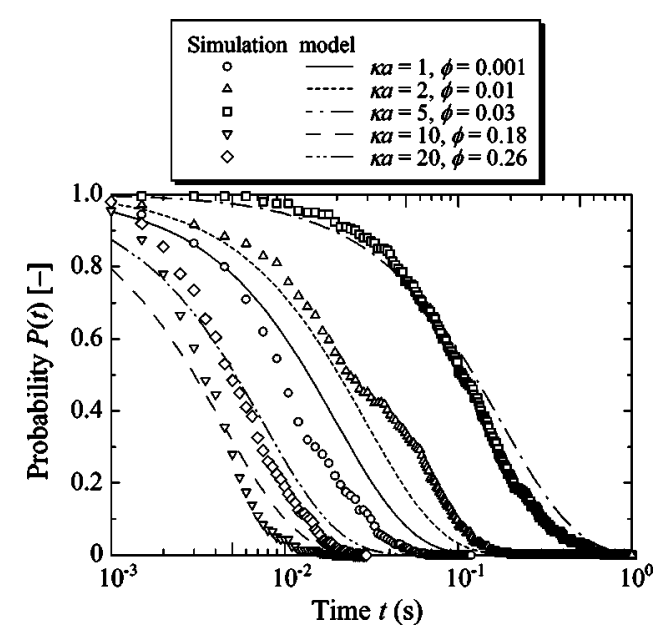

FIG. 11. Comparison of the probability for remaining in disordered state obtained from the present model with that from simulation results.

$$
\begin{aligned}
& c_{3}=\iint\left[w\left(\nu^{\mathrm{B}}\right) g\left(V_{\mathrm{b}}\right) J_{3}\right] d V_{\mathrm{b}} d \nu^{\mathrm{B}}, \\
& c_{4}=c_{4}^{\prime} \iint\left[w\left(\nu^{\mathrm{B}}\right) g\left(V_{\mathrm{b}}\right) J_{4}\right] d V_{\mathrm{b}} d \nu^{\mathrm{B}} .
\end{aligned}
$$

Here the distribution $g\left(V_{\mathrm{b}}\right)$ depends on $\kappa a$ and bulk concentration $\phi$, and the distribution for each condition of $\kappa a$ and $\phi$ was obtained by conducting the simulation of bulk dispersion phase with the periodic boundary condition in all directions.

Figure 11 compares the probability $P(t)$ obtained from the analytical model with that from the simulations. The parameter $c_{4}^{\prime}$ was determined so as to give the fit for the simulation result for $\kappa a=5, \phi=0.03$, and the value was about 10 and was applied to all other cases. It would be unavoidable for the parameter $c_{4}^{\prime}$ to involve some adjustable nature; the number of the disordered parts is difficult to count simply since the structure keeps fluctuating due to the Brownian motion. As an alternative, we examined the coordination number of each adsorbed particle, which would be an indication of the degree of disorder, and the number of particles with the coordination number other than 6 (i.e., 4, 5, 7, and 8) turned out to be 9.4 on average. The disordered parts would appear around these particles. The number of possible sites for the adsorption process may be slightly smaller than the number of the particle, 9.4 , but may well stay in the same order. Thus the value of the parameter $c_{4}^{\prime}$ would be reasonable. As seen in Fig. 11, the present model shows good accordance with the simulation results, which demonstrates that the model is quantitatively effective in describing the complicated probabilistic phenomena of the order formation with a rather simple concept. To see the importance of the successive processes we tried a model calculation only with the adsorption process, and confirmed that the single process model underestimates the probability $P(t)$ for smaller $\kappa a$. Thus, the accordance exemplifies the validity of the assumption that the last adsorption process is a successive process of the disturbance and adsorption processes.

\section{CONCLUSIONS}

We have simulated the colloidal adsorption and order formation of particles using a three-dimensional cell with an adsorptive substrate under a specified bulk concentration. Through examining the dynamics of order formation, the following conclusions are drawn.

(1) The order formation is shown to be a stochastic phenomenon; taking notice of the last adsorption that brings the transition from the structure with incomplete order to that with perfect order, the adsorption process is found to have an exponential-type distribution of the probability for the system remaining disordered. This result suggests that it is difficult to describe the time evolution of surface coverage or to expect the time taken to reach the ordered state in a deterministic way.

(2) Direct observation of the order formation process reveals that the process is composed of the disturbance process to make up a disordered squarelike part and the adsorption process into the disordered part. The two processes are successive, and a rate-determining step alters depending on the value of $\kappa a$.

(3) Based on the observation, a model to estimate the energy barrier for the order formation is developed, and the comparison of the model with the simulation result demonstrates that the model is quantitatively effective. The energy barrier obtained from the model is about $10 k_{\mathrm{B}} T$.

(4) A model to describe the probabilistic nature of the last adsorption process with time is developed and shown to be quantitatively valid by the comparison with the simulation result, which reinforces the basic mechanism of the order formation.

\section{ACKNOWLEDGMENTS}

This work was financially supported in part by the Grant-in-Aid for Scientific Research (B) from Japan Society for the Promotion of Science (JSPS), and by the New Energy and Industrial Technology Development Organization (NEDO) "Nanotechnology Program-Structuring Knowledge Project in Nanotechnology Materials Program" of the Ministry of Economy, Trade and Industry (METI).

${ }^{1}$ H. Hattori, Adv. Mater. (Weinheim, Ger.) 13, 51 (2001).

${ }^{2}$ O. D. Velev and E. W. Kaler, Langmuir 15, 3693 (1999).

${ }^{3}$ P. Hanarp, D. S. Sutherland, J. Gold, and B. Kasemo, Colloids Surf., A 214, 23 (2003).

${ }^{4}$ B. Kasemo, S. Johansson, H. Persson, P. Thormählen, and V. P. Zhdanov, Top. Catal. 13, 43 (2000).

${ }^{5}$ A. S. Dimitrov, T. Miwa, and K. Nagayama, Langmuir 15, 5257 (1999).

${ }^{6}$ P. A. Kralchevsky and K. Nagayama, Langmuir 10, 23 (1994).

${ }^{7}$ R. Micheletto, H. Fukuda, and M. Ohtsu, Langmuir 11, 3333 (1995).

${ }^{8}$ A. S. Dimitrov and K. Nagayama, Langmuir 12, 1303 (1996).

${ }^{9}$ C. A. Johnson and A. M. Lenhoff, J. Colloid Interface Sci. 179, 587 (1996).

${ }^{10}$ M. Semmler, E. K. Mann, J. Rička, and M. Borkovec, Langmuir 14, 5127 (1998).

${ }^{11}$ M. Semmler, J. Rička, and M. Borkovec, Colloids Surf., A 165, 79 (2000).

${ }^{12}$ D. A. Antelmi and O. Spalla, Langmuir 15, 7478 (1999).

${ }^{13}$ T. Serizawa, H. Takeshita, and M. Akashi, Langmuir 14, 4088 (1998).

${ }^{14}$ O. Spalla and S. Desset, Langmuir 16, 2133 (2000). 
${ }^{15}$ A. Thill and O. Spalla, Langmuir 18, 4783 (2002).

${ }^{16}$ D. W. Thompson and I. R. Collins, J. Colloid Interface Sci. 163, 347 (1994).

${ }^{17}$ P. Lavalle, A. L. DeVries, C.-C. C. Cheng, S. Scheuring, and J. J. Ramsden, Langmuir 16, 5785 (2000).

${ }^{18}$ J. J. Gray and R. T. Bonnecaze, J. Chem. Phys. 114, 1366 (2001).

${ }^{19}$ J. J. Gray and R. T. Bonnecaze, Langmuir 17, 7935 (2001).

${ }^{20}$ J. J. Gray, D. H. Klein, R. T. Bonnecaze, and B. A. Korgel, Phys. Rev. Lett. 85, 4430 (2000).

${ }^{21}$ J. J. Gray, D. H. Klein, B. A. Korgel, and R. T. Bonnecaze, Langmuir 17, 2317 (2001).
${ }^{22}$ M. Miyahara, S. Watanabe, Y. Gotoh, and K. Higashitani, J. Chem. Phys. 120, 1524 (2004).

${ }^{23}$ M. R. Oberholzer, N. J. Wagner, and A. M. Lenhoff, J. Chem. Phys. 107, 9157 (1997).

${ }^{24}$ P. González-Mozuelos and M. Medina-Noyola, J. Chem. Phys. 93, 2109 (1990).

${ }^{25}$ N. Kubota and T. Kawakami, J. Cryst. Growth 74, 259 (1986).

${ }^{26} \mathrm{E}$. J. W. Verwey and J. Th. G. Overbeek, Theory of The Stability of Lyophobic Colloids (Elsevier, Amsterdam, 1948).

${ }^{27}$ J. E. Sader, J. Colloid Interface Sci. 188, 508 (1997).

${ }^{28}$ D. L. Ermak, J. Chem. Phys. 62, 4189 (1975). 